\title{
A influência do sexo e da idade na prevalência de bolsas periodontais
}

\section{The influence of gender and age on the prevalence of periodontal pockets}

\author{
Luciana MACHION* \\ Patricia Moreira de FREITAS* \\ João Batista CESAR NETO** \\ Getúlio R. NOGUEIRA FILHO*** \\ Francisco H. NOCITI Jr. ${ }^{* * * *}$
}

\begin{abstract}
MACHION, L.; FREITAS, P. M.; CESAR NETO, J. B.; NOGUEIRA FILHO, G. R.; NOCITI Jr., F. H. A influência do sexo e da idade na prevalência de bolsas periodontais. Pesq Odont Bras, v. 14, n. 1, p. 33-37, jan./mar. 2000.

Este trabalho teve como objetivo avaliar a influência dos fatores de risco na prevalência de bolsas periodontais em pacientes atendidos na clínica do terceiro e quarto anos da Faculdade de Odontologia de Piracicaba - Unicamp. Foram avaliados 100 pacientes através do levantamento dos dados contidos nas fichas clínico-anamnésicas, sendo consideradas bolsas de profundidade: $3 \mathrm{~mm}, 5 \mathrm{~mm}, 7 \mathrm{~mm}$ e $10 \mathrm{~mm}$, de acordo com o sistema diagnóstico WS (SALLUM; SALLUM $^{27}$, 1996). Os resultados foram comparados entre as profundidades de sondagem e as variáveis idade, sexo, bem como sua distribuição por sextantes. Observou-se maior prevalência de bolsas periodontais no sexo masculino, bem como maior profundidade de sondagem em pacientes acima de 31 anos. A distribuição de bolsas periodontais entre os sextantes foi homogênea.
\end{abstract}

UNITERMOS: Bolsa periodontal; Prevalência; Fatores de risco.

\section{INTRODUÇÃO}

Estudos sobre a prevalência das doenças periodontais têm demonstrado que estas constituem um problema de saúde bucal. A gengivite é uma doença caracterizada pela inflamação do tecido gengival, que associada a perda de inserção é denominada periodontite ${ }^{3,16}$. Esta, em seus estágios mais avançados, é considerada uma das maiores responsáveis pela perda do órgão dental ${ }^{14,16,22}$. O início e progressão das infecções periodontais são claramente modificadas por condições sistêmicas e locais, que podem alterar a flora patogênica, denominadas fatores de risco. Estes devem ser identificados com o objetivo de reduzir a probabilidade de ocorrência da doença periodontal em uma população.

Estudos epidemiológicos têm demonstrado que a prevalência e severidade da doença periodontal se agrava com o aumento da idade ${ }^{3,4,15,16}$. Entretanto, as formas mais severas parecem estar mais evidente em adolescentes e jovens adultos do que em idosos $^{3}$. Segundo HUGSON; JORDAN ${ }^{16}$ (1982), em crianças e adolescentes gengivites são muito comuns, enquanto em adultos verifica-se um processo inflamatório envolvendo, além do periodonto de proteção, o periodonto de sustentação. Inúmeros trabalhos vêm confirmando que tanto gengivites como periodontites são mais freqüentes em homens do que em mulheres ${ }^{3,6,11,26}$, provavelmente em razão de uma pior higiene bucal ${ }^{3,30,31,33}$ e menor freqüência de visitas ao dentista ${ }^{3}$. RUSSEL ${ }^{26}$ (1957) comprovou que a doença periodontal quando presente em adolescentes tende a ser mais severa no sexo feminino, porém, após os 20-30 anos, apresenta-se mais severa entre os homens ${ }^{23}$.

O objetivo desse estudo foi avaliar a prevalência de bolsas periodontais através do sistema diagnóstico W. S., em pacientes atendidos na clínica da Faculdade de Odontologia de Piracicaba UNICAMP, identificando alguns fatores de risco associados a doença periodontal e elucidando a sua etiologia para, posteriormente, desenvolver procedimentos preventivos e terapêuticos.

\footnotetext{
*Alunas de Graduação; ** Estagiário; *** Doutor; **** Professor Doutor - Faculdade de Odontologia da Universidade de Campinas - Piracicaba.
} 
MACHION, L.; FREITAS, P. M.; CESAR NETO, J. B.; NOGUEIRA FILHO, G. R.; NOCITI Jr., F. H. A influência do sexo e da idade na prevalência de bolsas periodontais. Pesq Odont Bras, v. 14, n. 1, p. 33-37, jan./mar. 2000.

\section{MATERIAL E MÉTODO}

Um total de 100 fichas clínico-anamnésicas referentes a pacientes de periodontia das clínicas do terceiro e quarto anos da FOP-UNICAMP foram recolhidas para análise por dois pesquisadores, os quais catalogaram os dados pertinentes à pesquisa. As fichas foram preenchidas pelos próprios alunos de graduação no primeiro dia de consulta do paciente. A profundidade de bolsas periodontais presentes no periograma são de $3,5,7$ e $10 \mathrm{~mm}$, de acordo com o sistema WS (SALLUM; SALLUM $^{27}$, 1996). O sistema WS codifica as principais informações relativas ao aspecto gengival e grau de evolução da doença em cada sextante da dentição. De acordo com este sistema, anota-se na ficha clínica as profundidades de sondagem $3 \mathrm{~mm}$ (P1 - periodontite leve), $5 \mathrm{~mm}$ (P2 - periodontite moderada), $7 \mathrm{~mm}$ (P3 - periodontite avançada) e $10 \mathrm{~mm}$ (P4 - periodontite terminal). A sondagem foi realizada por sextantes e em quatro faces do dente - lingual, vestibular, mesial e distal. Não foi levada em consideração a quantidade de dentes por sextante. As fichas de pacientes que não apresentavam bolsas ou que haviam sido submetidos a cirurgia periodontal foram excluídos previamente.

A contagem de bolsas foi inicialmente realizada para todos os pacientes como um único grupo. As bolsas encontradas foram anotadas, levando-se em consideração a profundidade de sondagem e o sextante em que se localizavam. Posteriormente, duas variáveis foram consideradas para análise: gênero e idade.

As fichas foram separadas por gênero na sua totalidade, e uma nova contagem foi realizada. Para a análise da variável idade, foram considerados três grupos: grupo I - 15 a 30 anos; grupo II - 31 a 50 anos; e grupo III - acima de 50 anos; a contagem de bolsas foi realizada novamente para estes grupos.

Para ambas variáveis, consideradas como fatores de risco, foi considerada a distribuição das bolsas entre os sextantes. Para a expressão dos resultados foi realizada avaliação descritiva dos achados e então confeccionados gráficos percentuais dos valores totais encontrados em cada grupo e em cada variável isoladamente.

\section{RESULTADOS}

Foi encontrado um total de 3.634 bolsas periodontais. As bolsas de profundidade $3 \mathrm{~mm}$ foram as mais prevalentes $(57,3 \%)$, seguidas as de profundidade $5 \mathrm{~mm}(29,7 \%), 7 \mathrm{~mm}(11 \%)$ e $10 \mathrm{~mm}(2 \%)$. A distribuição das bolsas periodontais por sextantes foi relativamente uniforme (Gráfico 1).

Dos 100 pacientes analisados, 32 pertenciam ao sexo masculino e 68 ao sexo feminino. O gênero masculino deteve $36,7 \%$ das bolsas enquanto que o feminino, 63,3\%. A prevalência de bolsas periodontais foi ligeiramente maior no sexo masculino, como pode-se observar no Gráfico 2. A distribuição de bolsas por sextantes em ambos os sexos foi relativamente homogênea.

Nos três diferentes grupos etários, observou-se maior profundidade de sondagem a partir dos pacientes do grupo II, como mostra o Gráfico 3. Novamente, a distribuição de bolsas por sextantes foi uniforme no três grupos estudados.

\section{DISCUSSÃO}

Este estudo teve como objetivo verificar a influência dos fatores de risco (idade e gênero) na prevalência de bolsas periodontais em 100 pacientes atendidos na clínica de graduação da FOP-UNICAMP, levando-se em consideração a profundidade de sondagem e a distribuição das bolsas por sextante.

Foi observado neste trabalho uma alta prevalência de bolsas periodontais com profundidade $3 \mathrm{~mm}(57,3 \%)$ e, numa menor proporção, bolsas com profundidade $\geq 7 \mathrm{~mm}$ (13\%). BROWN ${ }^{6}$ (1990), avaliaram a prevalência de bolsas periodontais em adultos e verificou que bolsas de profundidade $7 \mathrm{~mm}$ eram raras $(0,6 \%)$ em todos os grupos etários analisados. Já STOLTENBERG ${ }^{29}$ (1993) verificaram, em uma amostra de 1.090 adultos, maior prevalência de bolsas $\geq 3 \mathrm{~mm}(45,5 \%)$ e menor prevalência de bolsas $\geq 5 \mathrm{~mm}(2,4 \%)$. Contudo, a menor prevalência de bolsas com profundidades

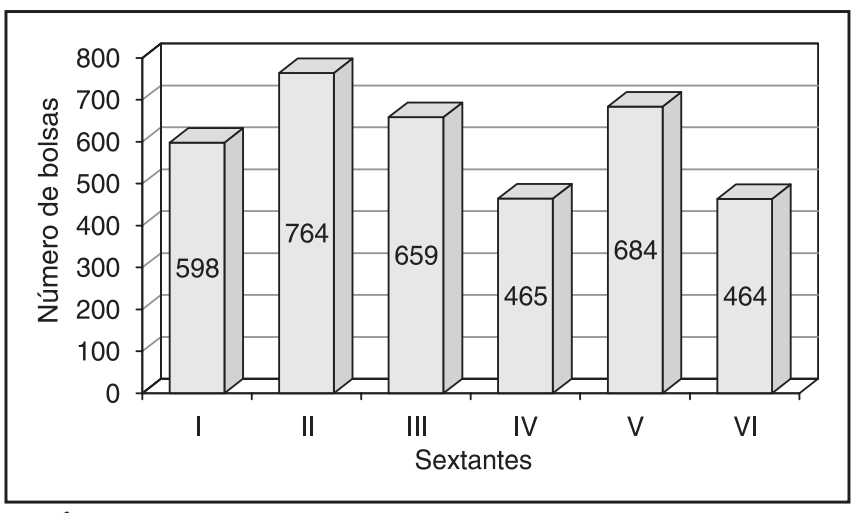

GRÁFICO 1 - Distribuição das bolsas periodontais por sextantes. 
MACHION, L.; FREITAS, P. M.; CESAR NETO, J. B.; NOGUEIRA FILHO, G. R.; NOCITI Jr., F. H. A influência do sexo e da idade na prevalência de bolsas periodontais. Pesq Odont Bras, v. 14, n. 1, p. 33-37, jan./mar. 2000.

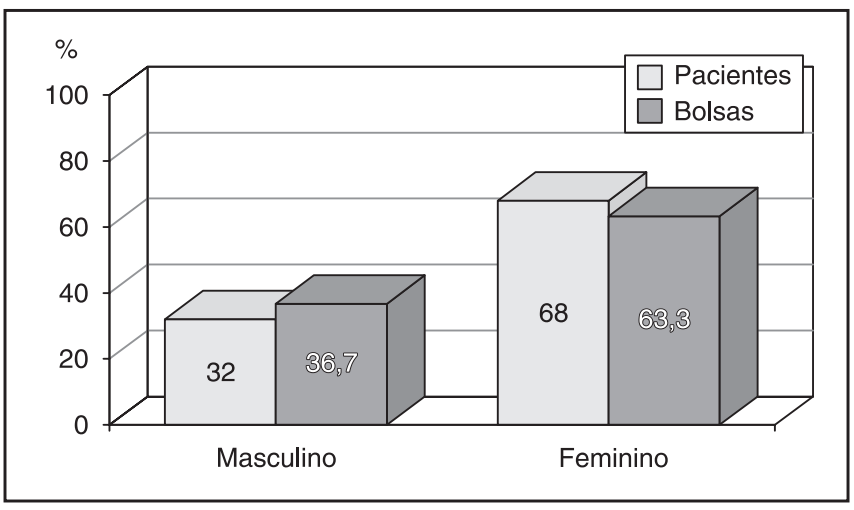

GRÁFICO 2 - Distribuição das bolsas periodontais por gênero.

$\geq 7 \mathrm{~mm}$, neste estudo, pode ser explicada pelo perfil dos pacientes atendidos na clínica de graduação da FOP-UNICAMP, onde os pacientes freqüentemente apresentam a ausência de dentes causada por doença periodontal severa ou cárie, fato este constatado por PAPAPANOU ${ }^{25}$ (1996), que reportou que a perda de dentes devido à severidade da doença periodontal pode subestimar valores epidemiológicos. A maior prevalência de bolsas $3 \mathrm{~mm}$ poderia ser justificada por vários fatores que devem ser levados em consideração para a avaliação da prevalência de bolsas periodontais: presença de bolsas falsas, sulcos profundos, presença de cálculo subgengival, dificuldade de sondagem nos sextantes posteriores e diferença de sondagem interexaminadores.

Quanto à distribuição de bolsas por sextantes, verificou-se uma prevalência relativamente uniforme das profundidades consideradas, em todos os grupos analisados, porém com ligeira diminuição da prevalência de bolsas nos sextantes IV e VI. Os estudos epidemiológicos, em sua maioria, verificaram a prevalência de bolsas periodontais por fa $\operatorname{ces}^{6,18,20}$, quadrantes ${ }^{18,31}$, ambos ${ }^{18,31}$, ou ainda em um sextante aleatório ${ }^{29}$. BAER; LESTER $^{5}$ (1988) sugerem a subestimação de valores epidemiológicos quando da avaliação de somente duas faces por dente em um quadrante superior e um inferior. Já BETHESDA ${ }^{31}$ (1987) e HUNT ${ }^{18}$ (1991) apresentaram resultados semelhantes para análises total e parcial de bolsas moderadas. Entretanto, para profundidades $\geq 4 \mathrm{~mm}$, os resultados foram subestimados em 13\%. Portanto, levando-se em consideração que a análise epidemiológica parcial ("partial mouth scores") da prevalência de bolsas periodontais pode subestimar os valores epidemiológicos ${ }^{8,18,29}$, este estudo avaliou a presença de bolsas de 3, 5, 7 e $10 \mathrm{~mm}$ em todas as faces de todos

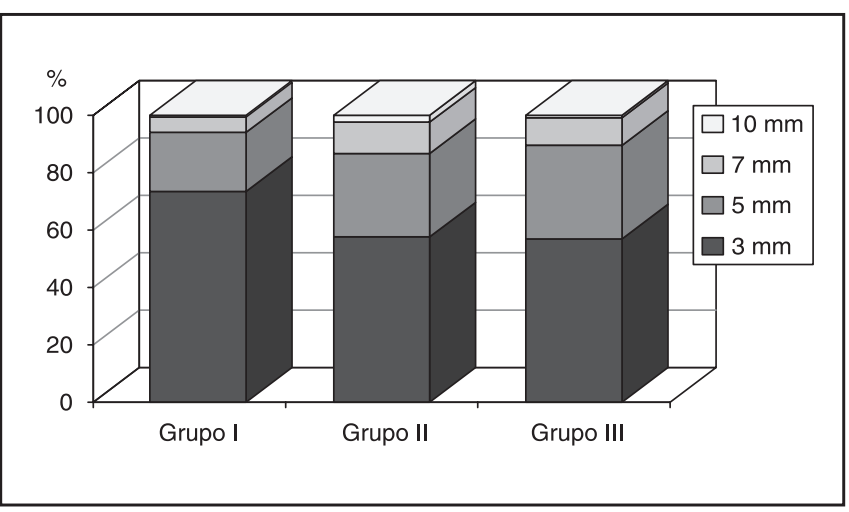

GRÁFICO 3 - Distribuição das bolsas periodontais por faixa etária.

os sextantes. No entanto, em função deste delineamento, a comparação dos dados epidemiológicos deste estudo com a literatura se torna limitada.

$\mathrm{Na}$ avaliação da prevalência de bolsas periodontais por grupo etário, verificou-se uma maior prevalência e aumento da profundidade de sondagem em indivíduos dos grupos II (31 a 50 anos) e III (acima de 50 anos). O grupo II apresentou maior prevalência que o grupo III, por ser o maior grupo dentre os três analisados (66\% dos pacientes). Este trabalho está de acordo com alguns estudos epidemiológicos encontrados na literatura ${ }^{4,16,26}$ onde a prevalência e a profundidade de bolsas periodontais aumentam com a idade. BROWN ${ }^{6}$ (1990) verificaram que a prevalência de bolsas periodontais no grupo de 18 a 24 anos era de $6 \%$ e que esta aumentava para mais de $18 \%$ no grupo de 55 a 64 anos. Em nosso estudo, verificou-se que a prevalência de bolsas no grupo I ( 15 a 30 anos) era de $9 \%$ e no grupo II (30 a 50 anos) houve um aumento de $23,7 \%$, proporcionalmente ao número de pacientes BETHESDA ${ }^{31}$ (1987) encontrou porcentagem de bolsas de profundidade $\geq 4 \mathrm{~mm}$ em $13,8 \%$ da população de 25 a 34 anos e de $53,6 \%$ na população de 55 a 64 anos; já bolsas $\geq 7 \mathrm{~mm}$ foram encontradas em 3,8\% do grupo de 35 a 44 anos e em $9,3 \%$ do grupo 55 a 64 anos. FOX $^{10}$ (1994) concluíram que individuos de 70 a 90 anos apresentam maiores indices de destruição periodontal, fato este que comprova as maiores profundidades de sondagem neste grupo. Estudos epidemiológicos ${ }^{12,24,28}$ comprovam que as bolsas de profundidade $\geq 6 \mathrm{~mm}$ são mais prevalentes em pessoas idosas. STOLTENBERG ${ }^{29}$ (1993) encontraram 39,5\% de bolsas $\geq 4 \mathrm{~mm}$ em pacientes de 30-34 anos e $65,7 \%$ em 65 a 69 anos. Esta prevalência pode ser explicada pelo tempo que os pacientes permaneceram susceptiveis à doença periodontal ${ }^{13}$. 
MACHION, L.; FREITAS, P. M.; CESAR NETO, J. B.; NOGUEIRA FILHO, G. R.; NOCITI Jr., F. H. A influência do sexo e da idade na prevalência de bolsas periodontais. Pesq Odont Bras, v. 14, n. 1, p. 33-37, jan./mar. 2000.

Devemos, entretanto, levar em consideração que a periodontite é uma doença de progressão lenta $^{16}$ e que o fator de risco idade parece ser cronológico. Assim, dentre a população idosa mais susceptivel à doença severa, a maioria possivelmente havia perdido os dentes ${ }^{3}$. Isto poderia explicar a baixa prevalência de severidade no terceiro grupo deste presente trabalho.

Quanto ao gênero, o sexo masculino apresentou maior prevalência de bolsas periodontais, estando de acordo com a literatura ${ }^{3,11,26}$. O sexo masculino deteve maior número de bolsas periodontais, quando comparados proporcionalmente ao sexo feminino, como se pode observar no Gráfico 2. O estudo de BROWN ${ }^{6}$ (1990) teve como resultado o aumento de 1,5 vezes no número de bolsas periodontais encontrado em pacientes do sexo masculino, em todas as faixas etárias analisadas. Os autores concluíram que uma amostra de donas de casa apresentam melhores condições periodontais do que homens da mesma idade. STOLTENBERG et al. ${ }^{29}$ (1993) não reportou diferenças entre ambos os sexos, embora tenha demonstrado maior profundidade de sondagem em homens. Os primeiros estudos feitos sobre a prevalência de bolsas periodontais em relação ao gênero demonstraram que a perda de inserção de todos os niveis de severidade são mais freqüentes no sexo masculino ${ }^{30,31,33}$. Os homens normalmente parecem apresentar uma pobre higiene bucal e maiores depósitos de cálculo e placa dental ${ }^{1,3,31,32}$. As razões para a diferença da condição periodontal entre homens e mulheres ainda não foram exploradas em detalhe, porém sugere-se que esta diferença estaria mais relacionada com a pobre higiene bucal dos homens ${ }^{3,11,27,32}$ e com sua menor freqüência ao dentista do que com qualquer outro fator genético ${ }^{3,27,32}$. CHEN; RUBINSON ${ }^{7}$ (1982), em uma amostra de 1.000 famílias americanas, reportaram semelhante freqüência de visitas ao dentista por adultos homens $(70,8 \%)$ e mulheres (79\%) num periodo de um ano. Este mesmo estudo, entretanto, verificou que $20 \%$ das mulheres entrevistadas faziam uso de fio dental diariamente, enquanto que o mesmo comportamento foi reportado por somente $11,5 \%$ dos homens.

Logo, parece evidente a existência de uma correlação entre a prevalência de bolsas periodontais com as variáveis estudadas. No entanto, devido às limitações e diferentes metodologias e populações inerentes aos estudos epidemiológicos, torna-se dificil o estabelecimento de uma relação direta de causa e efeito entre os fatores de risco e as bolsas periodontais. Portanto, são necessários mais estudos em âmbito nacional para uma melhor compreensão da prevalência da doença periodontal e a influência dos diversos fatores de risco relacionados a esta doença.

\section{CONCLUSÕES}

A partir deste trabalho, é pertinente afirmar que:

- foi observada uma alta prevalência de bolsas periodontais maiores que $5 \mathrm{~mm}$ na amostra avaliada de 100 pacientes da clínica da FOP-UNICAMP;

- houve uma distribuição uniforme de bolsas entre os sextantes, porém com ligeira diminuição na prevalência de bolsas nos sextantes IV e VI; - a prevalência de bolsas periodontais foi maior no gênero masculino do que no feminino;

- observou-se maior prevalência de bolsas e maior profundidade de sondagem em indivíduos acima de 31 anos.

MACHION, L.; FREITAS, P. M.; CESAR NETO, J. B.; NOGUEIRA FILHO, G. R.; NOCITI Jr., F. H. The influence of gender and age on the prevalence of periodontal pockets. Pesq Odont Bras, v. 14, n. 1, p. 33-37, jan./mar. 2000.

\begin{abstract}
The aim of this study was to evaluate the influence of risk factors on the prevalence of periodontal pockets in patients attending the clinic of the School of Dentistry of Piracicaba - Unicamp. The sample consisted of 100 patients attended by the students from the third and fourth years of undergraduation. The data were taken from anamneses and clinical - periodontal records. Probing depths were considered to be 3, 5, 7 and $10 \mathrm{~mm}$, according to the WS diagnosis system (SALLUM, 1996). The results were compared regarding pockets depths and the risk factors age and gender. Also, the distribution of pockets among the sextants was observed. This study indicated a major prevalence of periodontal pockets in men and an increase in probing depth in patients older than 31 years. The distribution of pockets among the sextants was relatively uniform in all studied groups.
\end{abstract}

UNITERMS: Periodontal pocket; Prevalence; Risk factors. 
MACHION, L.; FREITAS, P. M.; CESAR NETO, J. B.; NOGUEIRA FILHO, G. R.; NOCITI Jr., F. H. A influência do sexo e da idade na prevalência de bolsas periodontais. Pesq Odont Bras, v. 14, n. 1, p. 33-37, jan./mar. 2000.

\section{REFERÊNCIAS BIBLIOGRÁFICAS}

1. ABDELLATIF, H. M.; BURT, B. A. An epidemiological investigation into the relative importance of age and oral higiene status as determinants of periodontitis. J Dent Res, v. 66, p. $13-18,1987$

2. AINAMO, J.; AINAMO, A. Partial indices as indicators of the severity and prevalences of periodontal disease. Int Dent J, v. 35, p. 322-326, 1985.

3. Americam Academy of Periodontology: Epidemiology of Periodontal diseases. J Periodontol, v. 67, p. 935-945, Set., 1996.

4. American Academy of Periodontology: Section 1: Consensus report on periodontal diseases: epidemiology and diagnosis. J Periodontol, p. 216-222, 1996.

5. BAER, P. N.; LESTER, M. L. Oral health survey of US adults: NIDR 1985 national survey [editorial]. Periodontal Case Reports, v. 10, n. 7, 1988.

6. BROWN, L. J. Periodontal status of US employed adults in 1985-1986. JADA, v. 121, n. 2, p. 226-232, Agosto, 1990 .

7. CHEN, M. S.; RUBINSON, L. Preventive dental behavior in families: a national survey. J Am Dent Assoc, v. 105, p. 43-46, 1982.

8. DOWNER, M. C. The relative efficiencies of some periodontal partial recording selections. J Periodont Res, v. 15, p. 334-340, 1972.

9. FLEISS, J. L. Representativeness of the Ramfjord teeth for epidemiologic studies of gengivitis and periodontitis. Community Dent Oral Epidemiol, v. 15, p. 221-224, 1987.

10. FOX, C. H. Periodontal disease among New England elders.

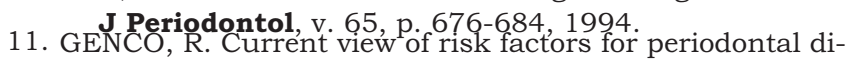
seases. J Periodontol, p. 1041-1049: Oct. 1996.

12. GILBERT, G. H.; HEFT, M. W. Periodontal status of older Floridians attending senior activity centers. J Clin Periodontol, v. 19, p. 249-255, 1992.

13. GRIFFITHS, G. S. et al. Detection of high risk groups and individuals for periodontal diseases. Clinical assessment of the periodontum. J Clin Periodontol, v. 15, p. 403-410, 1988.

14. JOHANSEN, J. R. A survey in Norway for causes of loss of permanent teeth and the number of teeth remaining after extraction. University of Oslo, 1970.

15. HUGSON, A. Prevalence and distribution of gengivitis-periodontitis in children and adolescents. Swed Dent J, v. 5, p. 91-103, 1981.

16. HUGSON, A.; JORDAN, T. Frequency distribution of individuals aged 20-70 years according to severity of periodontal disease. Community Dent Oral Epidemiol, v. 10, p. 187-192, 1982.

17. HUNT, R. J. The prevalence of periodontal attachment loss in an Iowa population aged 70 and older. J Public Health Dent, v. 50, p. 251-256, 1990.
18. HUNT, R. J, FANN, S. J. Effect of examining half the teeth in a partial periodontal recording of older adults. J Dent Res, v. 70, p. 1380-1385, 1991.

19. HUNT, R. J. The efficiency of half mouth examinations in estimating the prevalence of periodontal disease. $\mathbf{J}$ Dent Res, v. 66, p. 1044-1048, 1987.

20. KINGMAN, A. Systematic errors in estimating prevalence and severity of periodontal disease. J Periodontol, v. 59 , p. $707-713,1988$.

21. LOCKER, D.; LEAKE, J. L.: Periodontal attachment loss in independent living older adults in Ontario, Canada. J Public Health Dent, v. 53, p. 6-11, 1993.

22. MEHTA, F. S. Relative importance of the various causes of tooth loss. J All India Dent Assoc, v. 30, p. 211-221, 1958.

23. MEHTA, M. M. Periodontal disease among adults. Canad Den A J, v. 21, p. 617-624, Nov. 1955.

24. NORDERYD, O.; HUGSON, A. Risk of severe periodontal disease in a Swedish adult population. A cross sectional study. J Clin Periodontol, v. 25, n. 12, p. 1022-1028, Dec., 1998

25. PAPAPANOU, P. Section 1 A: Periodontal diseases - Epidemiology. J Periodontol Annals, p. 14-36, Set. 1996.

26. RUSSEL, A. L. Some epidemiological characteristics of periodontal disease in a series of urban populations. J Periodontol, p. 286-293, Dec. 1957.

27. SALLUM, A. W.; SALLUM, E. A. Indicação e Sequência em Cirurgia Periodontal. In: TODESCAN, F.; BOTTINO, M.: Atualização na clínica odontológica. A prática da clinica geral. Cap. 22, p. 585, 1996.

28. SLADE, G. D. Oral health status and treatment needs of noninstitutionalized person aged $60+$ in Adelaide, South Australia. Aust Dent J, v. 38, p. 373-380, 1993.

29. STOLTENBERG. Prevalence of periodontal disease in a health maintainence organization and comparisons to the national survey of oral health. J Periodontol, v. 64, p. 853-858, Sept, 1993.

30. U. S. Public Health Service, National Center for Health Statistics. Basic data on dental examination finding of person 1-74 years; United States, 1971-1974. DHEW Publ. No (PHS) 79-1662, Series 11 No. 214. Washington DC: Government Printing Office; 1979.

31. U.S. Public Health Service, National Institute of Dental research. Oral health of United States Adults; National findings. NIH Publ No 87-2868.; Bethesda, MD; NIDR, 1987.

32. U.S. Public Health Service, National Center for Health Statistics. Oral hygiene in adults, United States 1960-1962. PHS Publ, No 1000, series 11 No16. Washington DC: Government Printing Office; 1979.

33. U. S. Public Health Service, National Center for Health Statistics. Periodontal Disease in Adults, United States 1960-1962. PHS Publ. No. 1000, Series 11 No.12. Washington DC Government Printing Office; 1965. 